\title{
PERRIN Michel, Visions huichol. Un art amérindien du
}

Mexique

Éditions d'art Somogy, Paris, 2014

Yann Hutin

\section{CpenEdition}

\section{Journals}

Édition électronique

URL : https://journals.openedition.org/jsa/14334

DOI : 10.4000/jsa.14334

ISSN : 1957-7842

Éditeur

Société des américanistes

Édition imprimée

Date de publication : 31 décembre 2015

Pagination : 317-321

ISSN : 0037-9174

Référence électronique

Yann Hutin, « PERRIN Michel, Visions huichol. Un art amérindien du Mexique », Journal de la Société des américanistes [En ligne], 101-1 et 2 | 2015, mis en ligne le 15 mars 2016, consulté le 24 février 2023. URL : http://journals.openedition.org/jsa/14334 ; DOI : https://doi.org/10.4000/jsa.14334

Ce document a été généré automatiquement le 24 février 2023.

Tous droits réservés 


\section{PERRIN Michel, Visions huichol. Un art amérindien du Mexique}

Éditions d'art Somogy, Paris, 2014

Yann Hutin

\section{RÉFÉRENCE}

PERRIN Michel, Visions huichol. Un art amérindien du Mexique, Éditions d'art Somogy, Paris, 2014, 224 p., bibliogr., ill. coul., photos.

1 Visions huichol. Un art amérindien du Mexique présente un produit emblématique de la culture huichol ou wixárika selon l'ethnonyme autochtone. Sur un support en contreplaqué recouvert de résine sont appliqués des fils en laine ou en acrylique. Les motifs réalisés - personnages, artefacts, animaux, éléments naturels et traits ondulatoires - se caractérisent par la vivacité de leur couleur et leurs formes sinueuses. Ces tableaux de fil (cuadros de estambre en espagnol, yarn paintings en anglais) sont la projection d'un univers esthétique où se mêlent épisodes mythiques et accomplissements rituels. Cette convergence, fondamentale pour Michel Perrin qui considère que le rite est un mythe "en actes ", rend ainsi possible l'interprétation des œuvres selon l'un ou l'autre critère.

2 Les quelque cent vingt-cinq tableaux reproduits dans cet ouvrage d'art constituent une documentation iconographique exceptionnelle mise en valeur par une qualité d'édition particulièrement soignée. La richesse du contenu, qui regorge de précieuses informations sur la cosmologie, le système de croyances et les pratiques correspondantes, fait de Visions huichol un véritable objet ethnologique. L'exercice auquel se livre l'auteur est remarquable au regard du foisonnement de motifs qui rend les tableaux de fils complexes et difficiles à déchiffrer. Indice du souci pédagogique de Perrin, un chapitre est spécialement réservé à un « vocabulaire de signes » permettant au lecteur de s'orienter dans un écheveau symbolique dense, caractéristique d'un «art pictural» qui sollicite des actes de regard et de pensée. Chaque œuvre est ainsi 
accompagnée d'un commentaire détaillé avec, procédé hautement judicieux, l'addition de détails iconographiques à côté du texte. Ces pictogrammes, sélectionnés pour leur valeur significative, représentent les auxiliaires sémantiques idéals pour interpréter les tableaux.

Quatre axes thématiques charpentent l'ouvrage: l'art et la société, le mythe, le chamanisme et le rite. Le premier est développé dans les chapitres qui traitent de l'organisation sociale huichol, des modes d'expression artistique, de l'histoire des tableaux de fil, des techniques et des matériaux de fabrication employés, du répertoire symbolique figuré et du style iconographique. La "pensée mythique", quant à elle, s'exprime abondamment à travers les œuvres proposées et les commentaires qui relatent des épisodes primordiaux (l'apparition des ancêtres-animaux, le déluge, l'origine de l'agriculture, etc.) ou la naissance d'éléments naturels divinisés (le feu, le soleil, la lune, etc.). Exposés dans la première moitié du livre, ces thèmes sont suivis de la présentation d'un imago culturel considéré comme structurant - la «trilogie peyotlcerf-maïs »- et d'une figure souvent représentée dans les tableaux, une solanacée appelée kieri. Les chapitres qui leur sont consacrés annoncent celui réservé au chamanisme huichol dont ces éléments représentent certains attributs. Enfin, le quatrième axe aborde les rites du cycle agraire et cérémoniel (les sacrifices du cerf et du taureau en particulier) ainsi que les rituels du cycle vital (naissance, maladie et mort).

4 Les quatorze chapitres proposés, appelés «itinéraires", à l'image de ceux que le "chamane huichol [...] trace dans l'univers mythique»(p.14), témoignent de la cohérence de la démarche de Perrin, qui contextualise l'environnement social et symbolique de l'iconographie qu'il se propose d'examiner. Leur projection sur un plan esthétique est à l'origine du projet ethnologique qui anime l'ouvrage: «Quelles relations existe-t-il entre la religion et l'art d'une société traditionnelle? Comment un art pictural se nourrit-il d'un milieu géographique particulier et d'une manière spécifique de concevoir l'homme et le monde, exprimée par les mythes et les rites?» (p.9). Si l'auteur n'avance pas de réponses textuelles à ces questions ambitieuses formulées en incipit, c'est sans doute parce que les matériaux visuels qu'il présente agissent comme supports de la réflexion.

5 L'intitulé de l'ouvrage annonce les arguments développés au cours du livre. Le soustitre, Un art amérindien du Mexique, comporte des implications relatives au statut à accorder aux tableaux de fil. Perrin, fidèle à la perspective de deux pionniers de l'ethnologie huichol, Carl Lumholtz et Robert M. Zingg, qualifie les Huichol de « peuple d'artistes». Cette position, qui ouvre l'analyse des tableaux et en constitue le fondement, invite à ranger l'ensemble des productions matérielles huichol dans la catégorie de l'art. Une catégorie extensive au point d'inclure les instruments de musique, la broderie, les décorations corporelles, les arrangements floraux et les objets rituels. Sous cet angle-là, l'art des tableaux de fil apparaît comme le prolongement de l'ornementation des coupes votives sur lesquelles sont fixés, à l'aide de cire, des éléments (êtres humains, animaux, étoiles ou plants de maïs, par exemple) figurant des pétitions adressées aux divinités (p.16). Les pictogrammes utilisés dans les œuvres trouveraient, pour leur part, leur analogue morphologique et historique dans les motifs gravés sur des pierres cérémonielles ou sur des rochers (p. 49).

6 Quant au terme "amérindien", il entend souligner le caractère essentiellement indigène de la pratique. Cependant, le contexte historique, géographique et 
sociologique de l'apparition des tableaux de fil impose des limites à l'usage d'un tel adjectif. Dans l'introduction, le premier chapitre et la conclusion, l'auteur soulève, sans le qualifier de tel, le paradoxe qui consiste à définir comme traditionnelle une activité apparue dans les années 1950, d'impulsion extérieure, commerciale et exécutée principalement dans des foyers de peuplement éloignés des communautés indigènes. «[C]et art des tableaux de fil était pratiqué par un tout petit nombre. Il se réalisait surtout aux marges des communautés traditionnelles, dans de nouvelles urbanisations voisines des villes [et était] le fait d'artistes isolés encouragés par des ethnologues, des missionnaires et des fonctionnaires d'instituts indigénistes mexicains, véritables promoteurs d'un art trop vite qualifié de "chamanique" » (p. 9).

7 La portée des termes "art » et "amérindien », qualifiant les tableaux de fil, est par conséquent à nuancer si l'on tient compte des éléments de contexte disponibles. Insister sur leurs implications dans le cas huichol a pour intention de signaler une ambiguïté. Elle ne manque d'ailleurs pas d'apparaître dans le propos de l'auteur. Car, si ce dernier précise, à un moment donné, que « cet art devenu avant tout commercial a produit une sorte de renouveau culturel dans les communautés huichol qui se sont formées autour des zones urbaines » (p. 219), l'impression que donne la lecture du livre est que cette pratique artistique est un phénomène témoignant de l'acculturation et de la transformation de la tradition huichol dans son ensemble, alors qu'il n'est représentatif que d'une portion restreinte de la société.

8 Les observations qui précèdent ne fragilisent en rien l'argument principal de l'ouvrage exprimé, lui aussi, dans le titre : les tableaux de fil sont l'expression de "visions ». La question soulevée d'entrée est de savoir s'il s'agit d'un "art chamanique». Apparemment non. La plupart des artistes « reconnaissent illustrer la mythologie ou les visions décrites dans le chant des chamanes sans avoir de communication directe avec un monde-autre» (p.19). Ainsi, peu importe qu'il s'agisse d'un rêve ou d'une vision, ce qui compte pour l'artiste est la « dimension chamanique » de l'œuvre (ibid.). Perrin distingue d'ailleurs les productions de l'artiste «chamaniquement inspiré » de celles de l'artiste ordinaire. Le premier restituerait les messages qui lui auraient été communiqués lors d'un rituel alors que le second «illustrerait des récits » (ibid.). Cette distinction est à différencier de celle opérée par la suite à propos des «deux types extrêmes" de tableaux: d'un côté, ceux qui déploient une quantité importante de motifs "évoquant [...] la complexité des rites", de l'autre, ceux, plus sobres, qui expriment des relations entre des êtres (p. 46).

9 Au-delà du statut social du créateur, de ses intentions et de ses compétences, l'intérêt majeur, sur le plan ethnologique, réside dans le contenu de l'œuvre. L'iconographie du tableau de fil est caractéristique d'un «art de voir». Une question émerge alors : en quelle mesure la vision recherchée ou restituée par l'artiste, le sujet-créateur, se confond-elle avec celle de l'objet créé ? Rendre compte de cette relation au moyen de pictogrammes est assimilé à une incarnation de la vision de l'entité représentée, correspondant au phénomène de "voir de l'autre côté ", une disposition acquise par les initiés huichol appelée nierika ou «don de voir» - un concept qui qualifie d'ailleurs souvent les tableaux et s'y traduit par des cercles. "Ces forces invisibles sont parfois censées émaner de l'œuvre entière. Le tableau de fil ne serait plus alors une simple œuvre d'art mais un objet doté de pouvoirs comparables à ceux qu'il représente » (p. 49). 

conçoit que plusieurs sujets et objets habitent un tableau de fil et qu'ils sont par ailleurs impliqués dans des procès de métamorphose, l'«une des caractéristiques de l'art des tableaux » (p. 132), faisant de ceux-ci des images dotées de vie. L'identité ontologique des êtres représentés, en plus d'être instable, modifie la relation de communication qui s'engage entre l'artiste, son œuvre et les entités qui la peuplent. La complexité dialogique s'accroît encore dès lors qu'interviennent des références spatio-temporelles indexées dans l'iconographie par la représentation d'un mythe ou d'un rituel, avec une séquence d'actions perceptible et des déplacements dans l'espace. La lecture du tableau sollicite donc bien des actes de pensée, tributaires d'actes de regard qui parcourent l'œuvre selon l'agencement de pictogrammes fonctionnant comme des connecteurs logiques. Comme l'indique le commentaire d'un tableau représentant le sacrifice d'un taureau : « Tout ce qui se passe en bas agit en haut » (p. 199).

11 Au-delà de la compréhension du tableau déterminée par une opération iconique ou indexicale - dans le sens où la relation entre les signes distribués se fait selon la prise en compte du contexte propre à l'action représentée - la question de la nature de l'œuvre, en l'occurrence la transmutation de l'objet figuré en sujet agissant, intéressera de près l'ethnologie américaniste et l'anthropologie en général. Les modalités de communication dont le tableau est le vecteur sont révélatrices d'une pensée qui combine aspects concrets du monde et immatérialité des existants. Autrement dit, l'écriture pictographique «sert à entrer en contact avec l'invisible et même à le manipuler » (p. 49).

Visions huichol a le mérite d'ouvrir des pistes de recherche intéressantes. Précieux, il fournit au chercheur mésoaméricaniste un compendium de mythes et de rites déclinés sous une forme iconographique. Il représente également un compagnon de choix pour l'ethnographe qui saura mettre à profit ces ressources pour donner sens aux accomplissements rituels observables et s'interroger sur les dispositifs de communication entre des individus, des entités, des substances ou des principes ambulatoires. Car, il convient de le souligner, certains pictogrammes «méritent une attention particulière. Ils désignent non plus le visible, les êtres et les choses, mais des paroles, des forces, des dynamismes, des communications, des bruits, des "sécrétions" émanant du monde-autre ou bien provenant d'éléments de la nature, d'objets et d'êtres marqués par le sacré » (ibid.). Parallèlement, et cela intéressera l'anthropologie de l'art, les tableaux de fil invitent à s'interroger sur les mécanismes dont fait preuve une forme picturale pour traduire différents domaines d'expérience : le mythe, le rite, le politique, la technologie, les usages quotidiens ou encore les pratiques matrimoniales.

13 Les ethnologues trouveront dans ce livre une contribution indispensable à l'étude du symbolisme huichol. Et bien davantage encore, car l'analyse des cuadros de estambre menée par Perrin alimente la réflexion anthropologique contemporaine en ce qu'elle met en évidence un système de distribution ontologique indigène, traduit par le concept de vision et donné à voir sous la forme de récits pictographiques. Des dispositifs de mondiation y sont manifestés, au travers desquels le fond et la forme - sur les plans iconographique (sous-espaces vs motifs) et culturel (mythes vs rites) s'engendrent et se définissent mutuellement. Ils caractérisent ces œuvres dont l'originalité réside dans le fait que la technique et le style pictural révèlent des actes de communication qui articulent pratiques sociales, récits mythiques, création artistique et connaissance ésotérique.

Journal de la Société des américanistes, 101-1 et 2 | 2015 


\section{AUTEURS}

YANN HUTIN

Laboratoire d'anthropologie sociale (Las, EHESS), Paris 